\title{
Micro-Cloud Computing System by Human Quantum Computer
}

\section{J Ali ${ }^{1}$ and P P Yupapin ${ }^{2,3 *}$}

${ }^{1}$ Department of Physics, Faculty of Science, Universiti Teknologi Malaysia, Johor Bahru, Malaysia

${ }^{2}$ Advanced Studies Center, Department of Physics, King Mongkut's Institute of Technology Ladkrabang (KMITL), Thailand

${ }^{3}$ Quantum Life Institute, Sainoi, Nonthaburi, Thailand

World of small scale element (atom/particle) interaction can be successfully described by quantum mechanics, especially, photon or mass less particle behaviors which has been well defined. Mind (spirit) has been established as coherent light (photon), which is mass less. Mind behaviors can also be described by quantum mechanics. Spirit is localized and delocalized in brain by wave particle duality, where the body network is connected by spirit network, while the brain function is performed by the collapse of spirit wave form, i.e. projection operator known as consciousness (and sub-consciousness). This means that the projection of mind (spirit) can form the consciousness operator, where the brain and body (organs) can be functioned and transformed to the classical world. Brain signals (cytokines) are formed by coherent light and localized in 3 dimensions (holography) in human brain, where the superposition of particles (waves) have their own space-time geometry.

A paradox state of consciousness is a sub-consciousness state, which can be cloned (copied). The consciousness theory was proposed by many scientists [1-4], where the convincing theory of consciousness was given by Penrose [5]. The theory exposed by Penrose and his close colleague Hamer off is very detailed, where the story begins with Penrose's distinction between "subjective" and "objective" reduction [1]. Subjective reduction is what happens when an observer measures a quantity in a quantum system: the system is not in any specific state (the system is in a "super position" of possible states) until it is observed, in which the observation causes the system to reduce (or "collapse") to a specific state. This is the only reduction known to traditional quantum theory. Objective reduction is a Penrose discovery, where part of his attempt at unifying relativity theory and quantum theory. In his opinion, consciousness must be a quantum phenomenon because neurons are too big to account for consciousness. Inside neurons there is a "cytoskeleton", the structure that holds cells together, whose "micro-tubules" (hollow protein cylinders 25-nanometers in diameter) control the function of synapses. Penrose believes that consciousness is a manifestation of the quantum cytoskeletal state and its interplay between quantum and classical levels of activity. Under special circumstance, the system must then collapse to that one state. The collapse of the wave function is occurred, where the universe must choose between significantly differing space time geometry, for instance, the projection of brain signal to sensing cells. The link between brain signals and microtubules via cells can perform the neural (quantum) process, which is called the consciousness. These conformation states can interact with the neighboring states to represent, propagate and process information.

Human consciousness has become the key of investigations for cognitive scientists, where it is the key information for cognitive science study [6-8]. We know that it arises in the brain, but we don't know how or where in the brain, where it is exactly localized. We don't even know if it requires specialized brain cells (or neurons) or some sort of special circuit arrangement of them. Till date, nobody really knows what consciousness is, therefore, many scientists are seeking the way that consciousness to be the patterns of information, which can be transferred by electrons or photons, others will be able to copy it, edit it, sell it, or pirate it. It might be bundled with other information minds. The convincing concept can be categorized as the followings. String and spirit are the basic elements of matters and human beings in universe, respectively, where they are in the forms of coherent lights (photons), i.e. mass-less particles [9-11].

Regarding to quantum physics, the existence of photon in space and time is satisfied the uncertainty and paradox principles, which means that the measurement of consciousness can be realized by using the probe that can be connected to the basic element of consciousness, i.e. spirit or mind, where in this work the wave-particle duality probe (photon) is recommended to form the required measurement $[12,13]$. In principle, consciousness and sub-consciousness is the paradox each other, which can be formed by the paradox states between waking and sleeping (dreaming) situations, where the uncertainty principle between them is involved in the measurement process, which means that the measurement can be taken place, while the other information will be uncertained $[13,14]$. In applications, the consciousness and subconsciousness information can be experienced to be sensors such as color, smell, feeling, pleasure and fall in love, which will be useful for humanoid robotic applications $[15,16]$, which is the important issue of research and investigations nowadays.

In quantum physics, materials or non-materials can be existed and localized after projection (measurement and observation), otherwise, it is not existed. It is similar to Lord Buddha thought that human is not existed before the human thought forming. Consciousness and sub-consciousness are a paradox states each other, thus, they cannot be both localized exactly in the space-time geometry, which they can be localized in two different locations (paradox states). Mind (spirit) is formed by coherent light which is non-localized in 3 dimensions in brain. The projection of spirit (waves) can be connected to the required signals (sensors) under the consciousness situation, which is formed by sensor-cells-microtubules connection. Figure 1, the two quantum bits, $\alpha$ and $\beta$ tubulins, are processed and formed the operation within the microtubules (quantum processor) [17].

Figure 2 shows the schematic of cell and microtubule connection [17]. Cytokines are a broad and loose category of small proteins that are important in cell signaling, where they are released by cells and affect the behavior of other cells, and sometimes the releasing cell itself. In Figure 3, the quantum bit (Qubit) is operated and transferred to cells via microtubules, in which the resonant signals are tunneled

*Corresponding author: P P Yupapin, Advanced Studies Center, Department of Physics, Faculty of Science, King Mongkut's Institute of Technology Ladkrabang Bangkok (KMITL), Thailand; Tel: +66 02329 80; E-mail: kypreech@kmitl.ac.th

Received January 24, 2014; Accepted January 27, 2014; Published February 03, 2014

Citation: Ali J, Yupapin PP (2014) Micro-Cloud Computing System by Human Quantum Computer. J Biosens Bioelectron 5: e127. doi:10.4172/2155$6210.1000 \mathrm{e} 127$

Copyright: () 2014 Ali J, et al. This is an open-access article distributed under the terms of the Creative Commons Attribution License, which permits unrestricted use, distribution, and reproduction in any medium, provided the original author and source are credited. 
Citation: Ali J, Yupapin PP (2014) Micro-Cloud Computing System by Human Quantum Computer. J Biosens Bioelectron 5: e127. doi:10.4172/2155$6210.1000 \mathrm{e} 127$

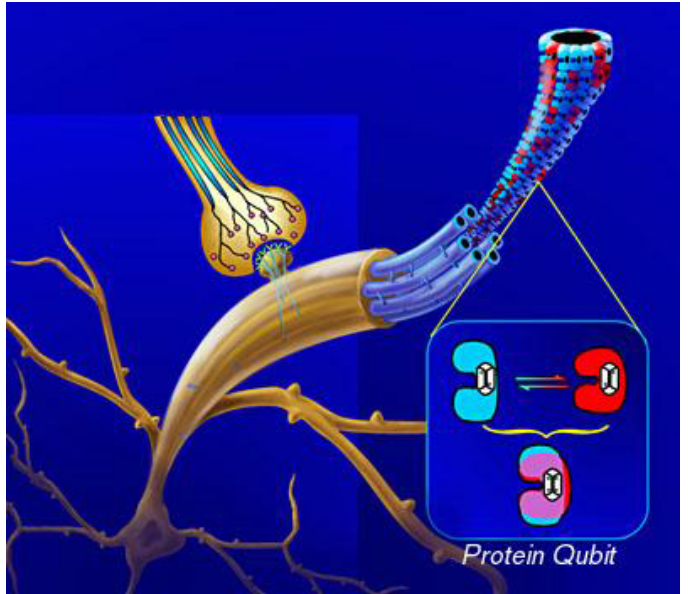

Figure 1: Microtubule schematic diagram, where two quantum bits ( $\alpha$ and $\beta$ tubulins) are generated and formed the link.

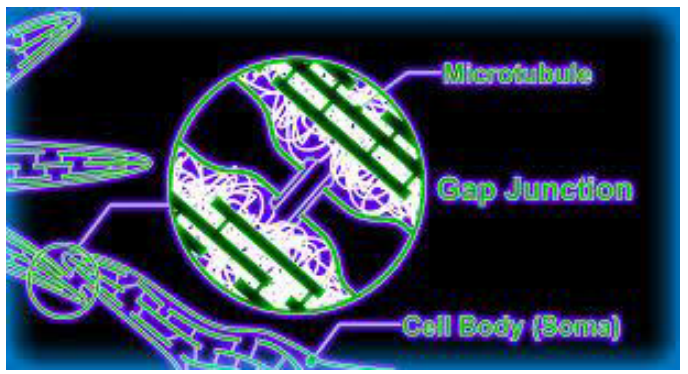

Figure 2: Cells and microtubule connection.

\section{Microtubule Schematic}

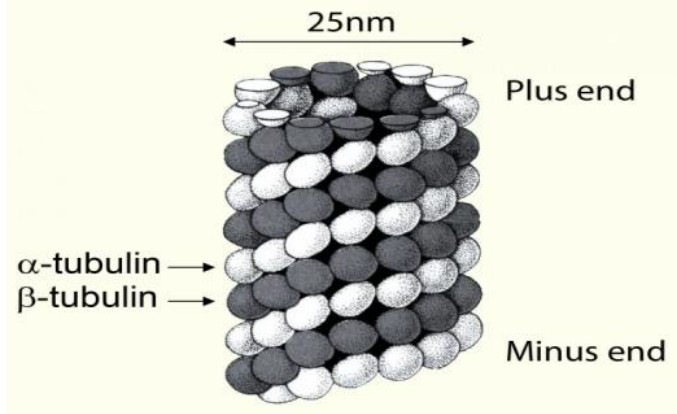

Figure 3: Quantum bit (Qubit) is operated and transferred to cells via microtubules.

and communicated with mind (modulated signals) and brain by the coherent light, where finally human network can be functioned [17]. The tunneling of spirit (coherent light) can be formed as shown in Figure 4, in which the Cerenkov radiation of the tunneling photon can be occurred $[17,18]$, the Whispering Gallery Mode (WGM) of wave can be generated [19]. In Figure 5, the projection of holographic request signals from the cloud computer into brain cells can be managed to link with the cells, cytokines, microtubules, in which the information can be obtained by the quantum computer [17].

The complete theoretical description of atom (particle or photon) where string is the basic element can begin with quantum mechanics,

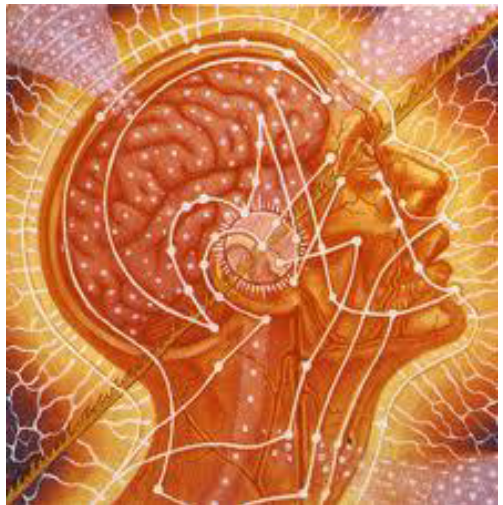

Figure 4: Tunneling and WGM of mind (spirit).

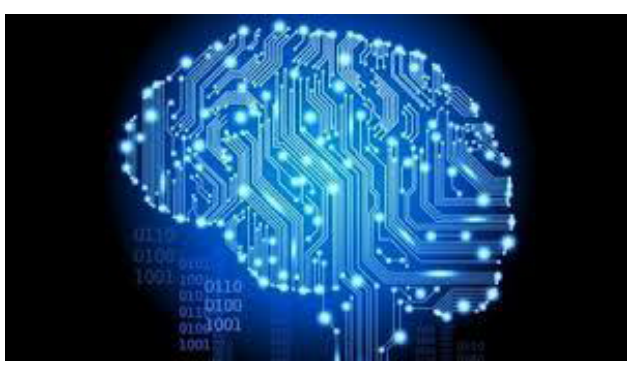

Figure 5: Brain central processor (Cloud computer)

special relativity and quantum field theory, where the distribution of force and field will spread entire the universe and galaxy, which can be described by a unified field theory(Grand unified field theory). The world of non-material which is formed by spirit (coherent light, mass less particle, photon) can be described by the same way but in this case the quantum of life (quantum life) is used to describe the spirit and its network spreading over the material region (organ and body), where the link between quantum field and quantum life will become the complete theory for the combination of them.

In conclusion, the quantum phenomenon of objective reduction controls the operation of the brain through its effects on coherent flows inside microtubules of the cytoskeleton. In general, the collapse of the wave function is what gives the laws of nature a non-algorithmic element. Otherwise we would simply be machines and we would have no consciousness, which is become a classical world. In a projection, the consciousness request is connected to the brain processor, in which all elements can be processed by a quantum processor, where finally the required information is transferred to the required destination by the quantum processor (microtubules) [20,21]. The emulation of brain computer (natural cloud computer) will be the big issue of current and future researches and investigations.

\section{Acknowledgment}

The authors would like to acknowledge the King Mongkut's Institute of Technology Ladkrabang (KMITL), Bangkok 10520, Thailand for giving the research facilities.

\section{References}

1. Hagan S, Hameroff SR, Tuszyn JA (2002) Quantum computation in brain microtubules: De-coherence and biological feasibility. Phys Rev E 65: $061901-$ $1-11$. 
Citation: Ali J, Yupapin PP (2014) Micro-Cloud Computing System by Human Quantum Computer. J Biosens Bioelectron 5: e127. doi:10.4172/2155$6210.1000 \mathrm{e} 127$

Page 3 of 3

2. Woolf NJ, Hameroff SR (2001) A quantum approach to visual consciousness. Trend in Cognitive Science 5: 472-478.

3. Bilodeau D (2014) Bohr's Mysticism. Journal of Consciousness Studies.

4. Winsberg E, Fine A (2003) Quantum life: Interaction, entanglement, and separation. Journal of Philosophy 100: 80-97.

5. Penrose R (2005) The Road to Reality: A complete guide to the laws of the universe. Vintage Books.

6. Cammarota JP (1991) Integrated System for detecting and managing acceleration-induced loss of consciousness. IEEE Engineering in Medicine and Biology Magazine 10: 52-55.

7. Choe Y, Kwon J, Chung JR (2012) Time, consciousness, mind uploading International Journal of Machine Consciousness 4:11-21.

8. Taylor JG (2009) Beyond consciousness. International Journal of Machine Consciousness 1:11-21.

9. Hall BC (2013) Quantum Theory for Mathematicians. Springer, New York.

10. Williams BO (2012) Study human transformation, physical and spiritual, Subtle Energy \& Energy Medicine, 19: 5-9.

11. McFarlane TJ (2002) Einstein and Buddha: The Parallel Sayings, Ulysses, USA.
12. Einstein A, Podolsky B, Rosen N (1935) Can quantum-mechanical description of physical reality be complete? Phys. Rev. 47: 777-780.

13. Yupapin PP, Ali J (2013) Cerenkov radiation: The space-time paradox. Nat \& Sci 11: 114-119.

14. McMahon D (2008) Quantum Field Theory. McGraw-Hill, New York.

15. Xie M (2010) Five steps of evolution from non-life to life-like robot. International Journal of Humanoid Robotics 6: 307-327.

16. Yokoi K, Kawauchi N, Sawasaki N, Nakashima T, Nakamura S, et al., (2004) Humanoid robotics applications in HRP. International Journal of Humanoid Robotics 1: 409-428.

17. Quantum consciousness (2014) Google search.

18. Georgescu J (2012) Cerenkov radiation: Light from ripples. Nature Physics 8 695-769.

19. Yupapin PP (2013) Nonlinear coupling effects of waves in a panda ring. Science Discovery 1: 1-5.

20. Yupapin PP, Pantian S, Ali J (2014) Novel design Rabi oscillation system for human quantum life detection probe. Life Sci J 11: 235-243.

21. Pantian S, Yupapin PP (2013) THz Rabi frequency oscillation for human consciousness/sub-consciousness detection probe use. J Biosensors and Bioelectronics 4: e126. 